\title{
Characterization of the sets of angular and global convergence, and of the sets of angular and global limits, of functions in a half-plane *
}

by

\section{F. Bagemihl and J. E. McMillan (Milwaukee)}

In the Euclidean plane provided with a Cartesian coordinate system, let $\mathcal{H}$ denote the upper half-plane and call the horizontal $x$-axis $R$. By an angle at a point $x \in R$ we mean a set of the form

$$
\Delta(x, \alpha, \beta)=\left\{x+r e^{i \theta}: 0<r<+\infty, a<\theta<\beta\right\}
$$

$$
(0<a<\beta<\pi, i=\sqrt{-1}) .
$$

We shall be concerned with functions $f$ that are defined and singlevalued in Je and assume finite real values. We call a point $x_{0} \in R$ a point of global convergence of $f$ provided that there exists a finite real number $y_{0}$ for which

$$
\lim _{\substack{z \rightarrow x_{0} \\ x \in \mathcal{J}}} f(z)=y_{0}
$$

$y_{0}$ is then termed the global limit of $f$ at $x_{0}$. A point $x \in R$ is called a point of angular convergence of $f$ provided that there exists a finite real number $y$ for which

$$
\lim _{\substack{z \rightarrow x \\ \varepsilon \in \Delta}} f(z)=y \quad \text { for every angle } \Delta \text { at } x ;
$$

$y$ is then termed the angular limit of $f$ at $x$. The set $A_{0}$ of all points of global convergence of $f$ will be called the set of global convergence of $f$, and the set $A$ of all points of angular convergence of $f$ will be referred to as the set of angular convergence of $f$. Then clearly $A_{0} \subset A$ (" $\subset$ " stands for set inclusion, not necessarily proper). If, for every $x \in A, \varphi(x)$ denotes the angular limit of $f$ at $x$, then $\varphi$ is a single-valued real-valued function

* Research supported by the National Science Foundation and the Wisconsin Alumni Research Foundation. 
defined on $A$, which we call the boundary function of $f$. We designate the set $\varphi(A)$ as the set of angular limits of $f$ and the set $\varphi\left(A_{0}\right)$ as the set of global limits of $f$.

In this setting, our theorems afford a complete characterization, for a function continuous in $\mathcal{H}$, of the sets of angular and global convergence, of the limit function, and of the sets of angular and global limits.

THEOREM 1. Let $f$ be an arbitrary real-valued function with domain 3 . Let $A_{0}$ be the set of global convergence, let $A$ be the set of angular convergence, and let $\varphi$ be the boundary function of $f$. Then $A_{0}$ is $a G_{\delta}, A$ is an $F_{\sigma \delta}$, $\varphi$ is of Baire class one on $A$ and continuous (relative to $A$ ) on $A_{0}$, and $A-A_{0}$ is a set of first category.

Proof. Throughout the proof, $k, m$ and $n$ denote natural numbers. Set

$$
S_{n}=\{x+i y:-\infty<x<+\infty, 0<y<1 / n\} .
$$

That $A_{0}$ is a $G_{\delta}$ is pointed ont by Hausdorff ([2], p. 275). We now prove that $A$ is an $F_{\sigma \delta}$. Set

$$
\Delta(x, n)=\Delta(x, 1 / n, \pi-1 / n) .
$$

For each $(k, m, n)$ the set $F_{k, m, n}$ of points $x \in R$ such that

$$
\left|f\left(z^{\prime}\right)-f\left(z^{\prime \prime}\right)\right|<1 / k \quad \text { if } \quad z^{\prime}, z^{\prime \prime} \in \Delta(x, n) \cap S_{m}
$$

is closed. Clearly

$$
A=\bigcap_{k, n} \bigcup_{m} F_{k, m, n}
$$

and so $A$ is an $F_{\sigma \delta}$

To prove that $\varphi$ is of the first class on $A$, it is sufficient to prove that for each real number $y$ each of the sets $\{x \in A: \varphi(x)>y\}$ and $\{x \in A: \varphi(x)<y\}$ is an $F_{\sigma}$ relative to $A$ ([2], p. 248). Let $y$ be a real number, and set $\Delta_{x}=\Delta(x, \pi / 4,3 \pi / 4)$. For each $(k, n)$ the set

$$
F_{k, n}=\left\{x: f\left(\Delta_{x} \cap S_{n}\right) \subset\left\{y^{\prime}: y^{\prime} \geqslant y+\frac{1}{k}\right)\right\}
$$

is closed. Thus since

$$
\left(\bigcup_{k, n} F_{k, n}\right) \cap A=\{x \in A: \varphi(x)>y\}
$$

the set $\{x \in A: \varphi(x)>y\}$ is an $F_{\sigma}$ relative to $A$. Similarly, $\{x \in A: \varphi(x)<y\}$ is an $F_{\sigma}$ relative to $A$.

It is clear that $\varphi$ is continuous on $A_{0}$, and the fact that $A-A_{0}$ is of first category follows from a theorem of Collingwood ([1], p. 1241, Theorem 4). Thus the proof of Theorem 1 is complete.
THeorem 2. Let $A_{0}$ be a $G_{\delta}$ in $R$, let $A$ be an $F_{\sigma \delta}$ containing $A_{0}$, and let $\varphi$ be a real-valued function of Baire class one on $A$ that is continuous (relative to $A$ ) on $A_{0}$. Then there exists a function $f$, real-valued and continuous in Je such that

(1) $A$ is the set of angular convergence and $\varphi$ is the boundary function of $f$ and

(2) at each $x \in A_{0}$, $f$ has the global limit $\varphi(x)$.

Moreover, if, in addition, $A-A_{0}$ is a set of first category, then there exists a function $f$, real-valued and continuous in 3 , such that (1) and

(3) $A_{0}$ is the set of global convergence of $f$.

Proof. We assume the hypotheses of the theorem except that we at present do not assume that $A-A_{0}$ is a set of first category.

We first prove the following

LEMMA. Let $U^{\prime}$ be an open set, and let $H^{\prime}$ be an $F_{\sigma}$ such that

$$
A_{0} \subset U^{\prime} \subset H^{\prime} \text { and } A \subset H^{\prime} \text {. }
$$

Let $\psi$ be a function of the first class on $A$ that is continuous (relative to $A$ ) on $A_{0}$, and let $\varepsilon$ be a positive number. Then there exist an open set $U$ containing $A_{0}$, an $F_{\sigma}$ set $H$ containing $U \cup A$, and a function $\Psi$ defined on $H$ such that

$$
U \subset U^{\prime}, \quad H \subset H^{\prime},
$$

$\Psi$ is of the first class on $H$ and is continuous on $U$, the range of $\Psi$ on $H-U$ is an isolated set, and

$$
|\Psi(x)-\psi(x)|<\varepsilon \quad \text { if } \quad x \in A .
$$

Proof of the lemma. For each $x \in A_{0}$, let $I_{x}$ be an open interval with midpoint $x$ such that the diameter of $\psi\left(I_{x} \cap A\right)$ is less than $\varepsilon / 4$. Set

$$
\begin{aligned}
& U=\left(\bigcup_{x \in A_{0}} I_{x}\right) \cap U^{\prime}, \\
& \Psi^{*}(x)=\sup \left\{\psi\left(x^{\prime}\right): I_{x^{\prime}} \ni x\right\} \quad(x \in U), \\
& \Psi_{*}(x)=\inf \left\{\psi\left(x^{\prime}\right): I_{x^{\prime}} \ni x\right\} \quad(x \in U) .
\end{aligned}
$$

Then $\Psi^{*}$ is lower semi-continuous and $\Psi_{*}$ is upper semi-continuous (on $U$ ). Let $\Psi$ be a continuous function on $U$ such that

$$
\Psi_{*}(x) \leqslant \Psi(x) \leqslant \Psi^{*}(x) \quad(x \in D)
$$

([2], p. 248). By a simple calculation we find that

$$
|\Psi(x)-\psi(x)|<\varepsilon \quad \text { if } \quad x \in U \cap A .
$$


Let $\mathfrak{J}$ be the family of open intervals with rational endpoints and length less than $\varepsilon / 4$. Since $\psi$ is of the first class on $A$, each of the sets

$$
\psi^{-1}(I)=\{x \in A: \psi(x) \in I\} \quad(I \in \mathfrak{J})
$$

is an $F_{\sigma}$ relative to $A$ ([2], p. 248). For each $I \in \mathcal{J}$, let $H_{I}$ be an (absolute) $F_{\sigma}$ such that

Set

$$
H_{I} \cap A=\psi^{-1}(I)
$$

$$
H^{*}=\left(\bigcup_{I \in \mathcal{J}} H_{I}\right) \cap H^{\prime} .
$$

Then $H^{*}$ is an $F_{\sigma}$. Let $a_{I}$ denote the midpoint of the interval $I$, and set

$$
\begin{array}{ll}
\psi^{*}(x)=\sup \left\{a_{I}: H_{I} \ni x, I \in J\right\} & \left(x \in H^{*}\right), \\
\psi_{*}(x)=\inf \left\{a_{I}: H_{I} \ni x, I \in J\right\} & \left(x \in H^{*}\right) .
\end{array}
$$

Then in the notation of [2], p. 235, $\psi^{*}$ is of class $\left(F_{\delta}, *\right)$, and $\psi_{*}$ is of class $\left(*, G_{\delta}\right)$. Thus $([2]$, pp. 242,243$)$ there exists a function $\hat{\psi}$ of the first class on $H^{*}$ such that

$$
\psi_{*}(x) \leqslant \hat{\psi}(x) \leqslant \psi^{*}(x) \quad\left(x \in H^{*}\right) .
$$

By a simple calculation,

$$
|\hat{\psi}(x)-\psi(x)| \leqslant 3 \varepsilon / 4 \quad \text { if } \quad x \in A .
$$

Let $\tilde{\psi}$ be a function of the first class on $H^{*}$ that has an isolated range and satisfies

([2], p. 247). Then

$$
|\widetilde{\psi}(x)-\hat{\psi}(x)|<\varepsilon / 4 \quad\left(x \in H^{*}\right)
$$

$$
|\widetilde{\psi}(x)-\psi(x)|<\varepsilon \quad \text { if } \quad x \in A .
$$

Let $H=H^{*} \cup U$, and extend the definition of $\Psi$ to all of $H$ by

$$
\Psi(x)=\widetilde{\psi}(x) \quad \text { if } \quad x \in H-U .
$$

Since a function is of the first class if and only if the preimage of each open set is an $F_{\sigma}$, it follows easily that $\Psi$ is of the first class on $H$. Since $\Psi$ clearly has the desired properties, the proof of the lemma is complete.

Let $\left\{U_{n}^{*}\right\}$ be a sequence of open sets, and let $\left\{H_{n}^{*}\right\}$ be a sequence of sets $F_{\sigma}$ such that $U_{n}^{*} \subset H_{n}^{*}$,

$$
A_{0}=\bigcap_{n=1}^{\infty} U_{n}^{*} \quad \text { and } \quad A=\bigcap_{n=1}^{\infty} H_{n}^{*} .
$$

We now define sequences $\left\{U_{n}\right\},\left\{H_{n}\right\}$ and $\left\{\varphi_{n}\right\}$ inductively. Applying the lemma, we let $U_{1}$ be an open set, let $H_{1}$ be an $F_{\sigma}$, and let $\varphi_{1}$ be a function defined on $H_{1}$ such that

$$
\begin{aligned}
A_{0} \subset U_{1} \subset H_{1}, & A \subset H_{1}, \\
U_{1} \subset U_{1}^{*}, & H_{1} \subset H_{1}^{*},
\end{aligned}
$$

$\varphi_{1}$ is of the first class on $H_{1}$ and continuous on $U_{1}$, the range of $\varphi_{1}$ on $H_{1}-U_{1}$ is isolated, and

$$
\left|\varphi_{1}(x)-\varphi(x)\right|<\frac{1}{4^{3}} \quad \text { if } \quad x \in A .
$$

Suppose now that $U_{j}, H_{j}$ and $\varphi_{j}$ are defined for $j=1, \ldots, n-1(n>1)$ so that $(j=1, \ldots, n-1)$

(7) $\varphi_{j}$ is a function of the first class on $H_{j}$ that is continuous on $U_{j}$,

and

$$
\begin{gathered}
U_{j} \text { is an open set, } H_{j} \text { is an } F_{\sigma}, \\
A_{0} \subset U_{j} \subset H_{j}, \quad A \subset H_{j}, \\
U_{j} \subset U_{j}^{*}, \quad H_{j} \subset H_{j}^{*},
\end{gathered}
$$

the range of $\varphi_{j}$ on $H_{j}-U_{j}$ is isolated,

$$
\left|\varphi(x)-\sum_{k=1}^{j} \varphi_{k}(x)\right|<\frac{1}{4^{j+2}} \quad \text { if } \quad x \in A
$$

for $j>1$ and $x \in H_{j}, \quad\left|\varphi_{j}(x)\right|<\frac{1}{4^{j}}$.

Then $\varphi-\sum_{j=1}^{n-1} \varphi_{j}$ is of the first class on $A$ and continuous (relative to $A$ ) on $A_{0}$. Thus, from the lemma, there exists an open set $U_{n}^{\prime}$ containing $A_{0}$, an $F_{\sigma}$ set $H_{n}^{\prime}$ containing ${J_{n}^{\prime}}^{\prime} \cup A$, and a function $\varphi_{n}$ defined on $H_{n}^{\prime}$, such that

$$
U_{n}^{\prime} \subset U_{n}^{*}, \quad H_{n}^{\prime} \subset H_{n}^{*},
$$

$\varphi_{n}$ is of the first class on $H_{n}^{\prime}$ and continuous on $U_{n}^{\prime}$, the range of $\varphi_{n}$ on $H_{n}^{\prime}-U_{n}^{\prime}$ is isolated, and

$$
\left|\varphi_{n}(x)-\left(\varphi(x)-\sum_{j=1}^{n-1} \varphi_{j}(x)\right)\right|<\frac{1}{4^{n+2}} \quad \text { if } \quad x \in A .
$$

It follows from (9) and (11) that

$$
\left|\varphi_{n}(x)\right|<\frac{1}{4^{n}} \quad \text { if } \quad x \in A .
$$

Fundamenta Mathematicae, T. LIX 
Set

and

$$
U_{n}=\left\{x \in U_{n}^{\prime}:\left|\varphi_{n}(x)\right|<\frac{1}{4^{n}}\right\}
$$

$$
H_{n}^{\prime \prime}=\left\{x \in H_{n}^{\prime}:\left|\varphi_{n}(x)\right|<\frac{1}{4^{n}}\right\} .
$$

Then $U_{n}$ is open, and since $H_{n}^{\prime \prime}$ is an $F_{\sigma}$ relative to the $F_{\sigma}$ set $H_{n}^{\prime}$, it is an (absolute) $F_{\sigma}$. Also, $A_{0} \subset U_{n}^{\prime} \cap A \subset U_{n}$, and $A \subset H_{n}^{\prime \prime}$. Set

$$
H_{n}=U_{n} \cup H_{n}^{\prime \prime} \text {. }
$$

Since $U_{n}^{\prime} \cap H_{n}^{\prime \prime} \subset U_{n}$, it follows that

$$
H_{n}-U_{n} \subset H_{n}^{\prime}-U_{n}^{\prime}
$$

and we see that the range of $\varphi_{n}$ on $H_{n}-U_{n}$ is isolated. Clearly,

$$
\left|\varphi_{n}(x)\right|<\frac{1}{4^{n}} \quad \text { if } \quad x \in H_{n} .
$$

Thus $U_{j}, H_{j}$ and $\varphi_{j}(j=1, \ldots, n)$ satisfy conditions (4) through (10) for $j=1, \ldots, n$.

We note that the above description works equally well for the case $n=2$, although statement $(10)$ is vacuous for this case. Thus we may suppose that we have $U_{j}, H_{j}$ and $\varphi_{j}(j \geqslant 1)$ defined so that for each $j$, statements (4) through (10) hold.

Observe that from (5) and (6) we have

$$
A_{0}=\bigcap_{n=1}^{\infty} U_{n}, \quad A=\bigcap_{n=1}^{\infty} H_{n} .
$$

For each open interval $I=\left(x_{1}, x_{2}\right)$ and each real number $t$ satisfying $0<t \leqslant 1$, define the cross cut $C(I, t)$ of $\mathcal{H}$ as follows. Set

$$
a=\frac{x_{2}+x_{1}}{2}+i t \frac{x_{2}-x_{1}}{2}
$$

and let $O(I, t)=C_{1} \cup C_{2}$, where $C_{j}$ is the shorter of the two arcs (including $a$ and excluding $x_{j}$ ) with endpoints $a$ and $x_{j}$ of the circle through $a$ that is tangent to $R$ at $x_{j}$. The definition of the modified cross cut $C_{n}(I, t)$ agrees with that of $C(I, t)$ except that whenever $x_{j} \notin H_{n}$, we replace $C_{j}$ by the rectilinear segment (including $a$ and excluding $x_{j}$ ) joining $a$ and $x_{j}$. Let $\Delta(I, t)$ and $\Delta_{n}(I, t)$ be the interiors of the Jordan curves $C(I, t) \cup \bar{I}$ and $C_{n}(I, t) \cup \bar{I}$, respectively (the bar denotes closure).

We now think of $n$ as fixed, and suppose for the sake of our notation that $U_{n}$ has infinitely many components; modifications for the case in which $U_{n}$ has only finitely many components will be obvious. Let $\left\{U_{n, j}\right\}_{j=1}^{\infty}$ be an enumeration of the components of $U_{n}$, and let $\left\{\boldsymbol{F}_{n, j}\right\}_{j=1}^{\infty}$ be a sequence of closed sets such that $F_{n, j} \subset F_{n, j+1}(j \geqslant 1)$ and

Set $F_{n, 1}^{\prime}=F_{n, 1}$

$$
H_{n}-U_{n}=\bigcup_{j=1}^{\infty} F_{n, j} \text {. }
$$

$$
F_{n, j}^{\prime}=F_{n, j} \cup\left[\bigcup_{k=1}^{j-1} \bar{U}_{n, k}\right] \quad(j>1),
$$

and let $\left\{V_{n, j, k}\right\}_{k}$ be an enumeration of the (possibly only finitely many) components of $R-F_{n, j}^{\prime}$. With each $V_{n, j, k}$ we associate a number $t_{n, j, k}$ such that

$$
0<t_{n, j, k} \leqslant \frac{1}{4}
$$

(13) the diameter of the circles in the definition of $O\left(V_{n, j, k}, 4 t_{n, j, k}\right)$ is greater than 1 ,

and

$$
\text { if } V_{n, j, k_{1}} \subset V_{n, j-1, k_{2}}(j>1) \text {, then } 4 t_{n, j, k_{1}}<t_{n, j-1, k_{2}} \text {. }
$$

Note that $U_{n, j} \cap F_{n, j}^{\prime}=\varnothing$, and let $k_{n, j}$ be the natural number such that, with the notation

$$
V(n, j)=V_{n, j, k_{n, j}}
$$

it is the case that $U_{n, j} \subset V(n, j)$. If $U_{n, j}$ and $V(n, j)$ have an endpoint $x_{0}$ in common, then $x_{0} \in F_{n, j}$, and thus $x_{0} \in H_{n}$. It follows that there exists a positive number $t_{n, j}$ such that $\left(t_{n, j} \leqslant 1\right)$

$$
C_{n}\left(U_{n, j}, t_{n, j}\right) \subset \Delta\left(V(n, j), t_{n, j, k_{n}, j}\right) .
$$

For each $V_{n, j, k}$, set

$$
f_{n}(z)=\left\{\begin{array}{lll}
0 & \text { if } & z \in C\left(V_{n, j, k}, 3 t_{n, j, k}\right), \\
\frac{1}{4^{n}} & \text { if } & z \in C\left(\nabla_{n, j, k}, 2 t_{n, j, k}\right)
\end{array}\right.
$$

and for each $U_{n, j}$, set $(z=x+i y)$

$$
f_{n}(z)=\left\{\begin{array}{ccc}
0 & \text { if } & z \in C_{n}\left(U_{n, j}, \frac{3}{4} t_{n, j}\right), \\
1 / 4^{n} & \text { if } & z \in C_{n}\left(U_{n, j}, \frac{1}{2} t_{n, j}\right), \\
\varphi_{n}(x) & \text { if } & z \in \bar{\Delta}\left(U_{n, j}, \frac{1}{4} t_{n, j}\right) \cap \nVdash .
\end{array}\right.
$$

Since $F_{n, j-1}^{\prime} \subset F_{n, j}^{\prime}(j>1)$, it follows from (14) that definition (16) is possible; and from (15) we see that (17) is compatible with (16). At this point we have defined $f_{n}(z)$ on a set which is closed relative to $J e$ and is contained in the open set

$$
\Delta_{n}=\left[\bigcup_{i, k}\left\{\Delta\left(V_{n, j, k}, 4 t_{n, j, k}\right)-\bar{\Delta}\left(V_{n, j, k}, t_{n, j, k}\right)\right\}\right] \cup\left[\bigcup_{j} \Delta_{n}\left(U_{n, j}, t_{n, j}\right)\right] .
$$


We now suppose that the range of $\varphi_{n}$ on $H_{n}-U_{n}$ is an infinite set; modifications for the case in which it is finite will be obvious. Let $\left\{a_{n, j}\right\}_{j=1}^{\infty}$ be an enumeration of the range of $\varphi_{n}$ on $H_{n}-U_{n}$. Since the range of $\varphi_{n}$ on $H_{n}-U_{n}$ is isolated, for each $(n, j)$ the set

$$
H_{n, j}=\left\{x \in H_{n}-U_{n}: \varphi_{n}(x)=a_{n, j}\right\}
$$

is an $F_{\sigma}$ relative to the $F_{\sigma}$ set $H_{n}-U_{n}$, and is therefore an (absolute) $F_{\sigma}$. Let $\left\{F_{n, j, k}\right\}_{k=1}^{\infty}$ be a sequence of closed sets such that

$$
F_{n, i, k} \subset F_{n, j, k+1} \quad(k \geqslant 1)
$$

and

$$
H_{n, j}=\bigcup_{k=1}^{\infty} F_{n, j, k} \text {. }
$$

Then for $(n, k)$ fixed, $\left\{F_{n, j, k}\right\}_{j=1}^{k}$ is a pairwise disjoint family of closed sets, and we can find a positive number $r_{n, k}$ such that $r_{n, k}<1 / k$ and, with the notation

$$
S_{n, j, k}=\bigcup_{x \in F_{n}, j, k}\left\{\zeta:\left|\zeta-\left(x+i r_{n, k}\right)\right| \leqslant r_{n, k}\right\} \quad(j=1, \ldots, k),
$$

it is the case that $\left\{S_{n, j, k}\right\}_{j=1}^{k}$ is a pairwise disjoint family of closed sets. For each $(n, j, k)(j \leqslant k)$ set

$$
f_{n}(z)=a_{n, j} \quad \text { if } \quad z \in S_{n, j, k} \cap\left(\mathcal{H}-\Delta_{n}\right) .
$$

By (16), (17), and (19), $f_{n}$ is defined on a set $S_{n}$ that is closed relative to $H$, and $f_{n}$ is continuous on $S_{n}$. From (10), (16), (17), and (19),

$$
\left|f_{n}(z)\right| \leqslant \frac{1}{4^{n}} \quad\left(z \in S_{n}, n>1\right) .
$$

Thus, by Tietze's theorem, $f_{n}$ has a continuous èxtension $f_{n}$ to all of $H_{\text {; }}$ and in the case $n>1$, we can require that

$$
\left|f_{n}(z)\right| \leqslant \frac{1}{4^{n}} \quad(z \in \mathcal{H}, n>1) .
$$

Clearly,

(21) if $x \in U_{n}$, then $f_{n}$ has the global limit $\varphi_{n}(x)$ at $x$.

Suppose now that $x \in H_{n}-U_{n}$. From (13), if $x \notin V_{n, j, k}$, then

$$
\Delta\left(\nabla_{n, j, k}, 4 t_{n, j, k}\right) \cap\{\zeta:|\zeta-(x+i)|<1\}=\varnothing .
$$

Keeping $n$ fixed, we note that $x$ is in only finitely many of the sets $V_{n, j, k}$. Thus, in particular, we have from (15) that for all sufficiently large $j$,

$$
\Delta_{n}\left(U_{n, j}, t_{n, j}\right) \cap\{\zeta:|\zeta-(x+i)|<1\}=\emptyset .
$$

Hence, for some sufficiently small positive number $h$,

$$
\Delta_{n} \cap\{\zeta:|\zeta-(x+i h)|<h\}=\varnothing .
$$

We see from (18) that for some sufficiently large $k$,

$$
x \in \bigcup_{j=1}^{k} F_{n, j, k} .
$$

Thus it follows from (19) that

(22) if $x \in H_{n}$, then $f_{n}$ has the angular limit $\varphi_{n}(x)$ at $x$.

Suppose now that $x \notin H_{n}$. If $x \in \bigcup_{j=1}^{\infty} \bar{U}_{n, j}$, then it follows from the definition of $C_{n}(I, t)$ and (17) that there exists an angle at $x$ in which the oscillation of $f_{n}$ at $x$ is at least $1 / 4^{n}$. If $x \notin \bigcup_{j=1}^{\infty} \bar{U}_{n, j}$, then for each $j$, $x \notin F_{n, j}^{\prime}$, and there exists $k_{j}$ such that $x \in \nabla_{n, j, k_{j}}$. Thus from (14) and (16), $f_{n}$ has an oscillation at $x$ of at least $1 / 4^{n}$ in each angle at $x$. Hence,

(23) if $x \notin H_{n}$, then there exists an angle at $x$ in which the oscillation of $f_{n}$ at $x$ is at least $1 / 4^{n}$.

Set

$$
f(z)=\sum_{n=1}^{\infty} f_{n}(z) \quad(z \in \mathcal{H}) .
$$

From $(20)$ we obtain $(m \geqslant 1, z \in \mathcal{H})$

$$
\sum_{n=m+1}^{\infty}\left|f_{n}(z)\right| \leqslant \frac{1}{3} \cdot \frac{1}{4^{m}} ;
$$

in particular, $f$ is continuous in $\mathfrak{H}$.

It is clear from (9), (12), (21), and (24) that (2) holds. Similarly it is clear from (9), (12), (22), and (24) that if $x \in A$, then $f$ has the angular limit $\varphi(x)$ at $x$. Suppose that $x \notin A$. We wish to prove that $f$ does not have an angular limit at $x$. Applying (12), let $m$ be the least natural number $n$ such that $x \notin H_{n}$. If $m>1$, we have from (22) that $\sum_{n=1}^{m-1} f_{n}$ has an angular limit at $x$. Thus from (23) and (24), there exists an angle at $x$ in which the oscillation of $f$ at $x$ is positive, and we have established (1).

We now assume that $A-A_{0}$ is a set of first category. Then $A-A_{0}$ is contained in an $F_{\sigma}$ set $H$ of first category. By considering the set

$$
U_{n} \cup\left[H \cap_{*}\left(H_{n}-U_{n}\right)\right],
$$


we see that we may suppose $H_{n}$ to have been chosen so that $H_{n}-U_{n}$ is a set of first category. Let $x \in A-A_{0}$, and let $m$ be the least natural number $n$ such that $x \notin U_{n}$. Then $x \in H_{m}-U_{m}$. Let $H_{m}^{\circ}$ denote the interior of $H_{m}$. Since

$$
H_{m}^{\circ}-\bar{U}_{m} \subset H_{m}-U_{m},
$$

the open set $H_{m}^{\circ}-\bar{U}_{m}$ is empty; in particular, $x \notin H_{m}^{\circ}-\bar{U}_{m}$. Thus, either $x$ is an accumulation point of $R-H_{m}$, or $x$ is an accumulation point of $U_{m}$. In the first case, it follows from (23) that the global oscillation of $f_{m}$ at $x$ is at least $1 / 4^{m}$. But from the definition of $m$ and (21), for $m>1, \sum_{n=1}^{m-1} f_{n}$ has a global limit at $x$. Thus from $(24), f$ does not have a global limit at $x$. In the second case, it follows from (17) and a similas argument that $f$ does not have a global limit at $x$, and we have established (3). This completes the proof of the theorem.

By taking $A_{0}=A$ or $A_{0}=\varnothing$ in Theorem 2, we obtain the following corollaries.

COROLLARY 1. Let $A_{0}$ be a $G_{\delta}$ in $R$, and let $\varphi$ be a continuous function on $A_{0}$. Then there exists a function $f$, continuous in $\mathcal{H}$, such that $A_{0}$ is the set of angular convergence as well as the set of global convergence of $f$, and $\varphi$ is the boundary function of $f$.

CoRollary 2. Let $A$ be an $F_{\sigma \delta}$ of first category in $R$, and let $\varphi$ be a function of Baire class one on $A$. Then there exists a function $f$, continuous in $\mathcal{H}$, such that $A$ is the set of angular convergence and $\varphi$ is the boundary function of $f$, and the set of global convergence of $f$ is empty.

THEOREM 3. Let $S$ and $T$ be sets of real numbers with $S \subset T$. Then $S$ and $T$ are analytic sets if and only if there exists a function $f$, real-valued and continuous in $\mathcal{H}$, such that $S$ is the set of global limits and $T$ is the set of angular limits of $f$.

Proof. Suppose first that $f$ is an arbitrary real-valued function with domain $\mathcal{H}$, and denote the sets of global and angular convergence of $f$ by $A_{0}$ and $A$, respectively. Then according to Theorem $1, A_{0}$ is a $G_{\delta}$ $A$ is an $F_{\sigma \delta}$, and the boundary function $\varphi$ of $f$ is of Baire class one on $A$. Since the Baire image of a Borel set is an analytic set ([2], p. 266), the sets $\varphi\left(A_{0}\right)$ of global limits of $f$ and $\varphi(A)$ of angular limits of $f$ are analytic.

Suppose now that $S$ and $T$ are analytic sets with $S \subset T$. We wish to show that there exists a function $f$, continuous in $J e$, having $S$ as its set of global limits and $T$ as its set of angular limits. This is obvious if $T=\varnothing$; so assume that $T \neq \varnothing$. Let $C$ be a perfect nowhere dense set of positive real numbers. Then there exists $([3]$, p. 388) a real-valued function $\tau(x)$ of Baire class one on $C$ such that

$$
\tau(C)=T
$$

Let $R_{-}$denote the set of non-positive real numbers. If $S \neq \varnothing$, let ([4], p. 82) $\sigma(x)$ be a real-valued function $R_{\text {- such that }}$

(26) $\sigma$ is continuous on the left at every point $x \in R_{-}$,

$$
\sigma\left(R_{-}\right)=S,
$$

(28) for every $y \in S$ there exists a point $x$ of continuity of $\sigma$ such that $\sigma(x)=y$.

Let $D$ be the set of points of discontinuity (including 0 ) of $\sigma$. Because of (26), $D$ is at most countable, and $\sigma$ is of Baire class one on $R_{-}$. Now let

$$
\begin{aligned}
& A_{0}=R_{-}-D, \quad A=C \cup R_{-} \quad \text { if } \quad S \neq \varnothing ; \\
& A_{0}=\varnothing, \quad A=C \quad \text { if } S=\varnothing \text {; } \\
& \varphi(x)=\left\{\begin{array}{lll}
\sigma(x) & \text { for } & x \in R_{-} \\
\tau(x) & \text { for } & x \in C
\end{array} \quad \text { if } \quad S \neq \varnothing ;\right. \\
& \varphi(x)=\tau(x) \quad \text { for } \quad x \in C \quad \text { if } \quad S=\emptyset .
\end{aligned}
$$

It is easily verified that all the hypotheses of Theorem 2 are satisfied, and so there exists a function $f$, real-valued and continuous in $x$, such that $A$ is the set of angular convergence, $A_{0}$ is the set of global convergence, and $\varphi$ is the boundary function of $f$. In view of (27) and (28), $S$ is the set of global limits of $f$, and (25) and (27) imply that $T$ is the set of angular limits of $f$. This completes the proof of the theorem.

CoRollary 3. A necessary and sufficient condition that a set $T$ of real numbers be an analytic set is that there exist a function $f$, real-valued and continuous in $\mathrm{Je}$, having $T$ as its set of angular limits.

Poprougénko has shown ([4], p. 82) that a necessary and sufficient condition that a non-empty set $T$ of real numbers be an analytic set is that there exist a harmonic function $f$ in He having $T$ as its set of global limits. It would be interesting to know whether this result remains valid with "global" replaced by "angular".

\section{References}

[1] E. T. Collingwood, Oluster sets of arbitrary functions, Proc. Nat. Acad. Sci. 46 (1960), pp. 1236-1242

[2] F. Hausdorff, Mengenlehre, 2d ed., Berlin-Leipzig 1927.

[3] C. Kuratowski, Topologie I, 2d ed., Mon. Matemat. 20, Warszawa-Wroclaw 1948.

[4] G. Poprougénk o, Sur l'analyticité des ensembles (A), Fund. Math. 18 (1932), pp. 77-84.

UNIVERSITY OF WISCONSIN, MILWAUKEE

Reģu par la Rédaction le 15.9.1965 